TIPA. Travaux interdisciplinaires sur la parole et le langage

37 | 2021

La convergence

\title{
La négation en berbère tasahlit : à propos des éléments de négation verbale d'étymons arabes
}

Negation in Tasahlit Berber: on elements of verbal negation of Arabic etymons

\section{Massinissa Garaoun}

\section{(2) OpenEdition}

1 Journals

Édition électronique

URL : https://journals.openedition.org/tipa/4253

DOI : 10.4000/tipa.4253

ISSN : 2264-7082

Éditeur

Laboratoire Parole et Langage

\section{Référence électronique}

Massinissa Garaoun, «La négation en berbère tasahlit : à propos des éléments de négation verbale d'étymons arabes », TIPA. Travaux interdisciplinaires sur la parole et le langage [En ligne], 37 | 2021, mis en ligne le 23 juillet 2021, consulté le 20 octobre 2021. URL : http://journals.openedition.org/tipa/4253 ; DOI : https://doi.org/10.4000/tipa.4253

Ce document a été généré automatiquement le 20 octobre 2021.

La revue TIPA. Travaux interdisciplinaires sur la parole et le langage est mise à disposition selon les termes de la licence Creative Commons Attribution - Pas d'Utilisation Commerciale - Pas de Modification 4.0 International. 


\title{
La négation en berbère tasahlit : à propos des éléments de négation verbale d'étymons arabes
}

Negation in Tasahlit Berber: on elements of verbal negation of Arabic etymons

\author{
Massinissa Garaoun
}

\section{Introduction ${ }^{1}$}

1 L'arabe et le berbère sont en contact dans le Maghreb occidental depuis plus de 1200 ans. Le berbère contemporain présente une multitude de variétés, marquées à différents degrés et niveaux par le contact avec l'arabe. Parmi celles-ci, le berbère tasahlit, pratiqué dans la Kabylie des Babors en Algérie, nous offre des données encore tout à fait inexplorées. En contact avec l'une des premières variétés d'arabe ayant émergé en Afrique du nord, cette langue berbère présente des phénomènes d'emprunt et de convergence profonds et complexes.

2 Dans cet article, la convergence est considérée comme un changement translinguistique caractérisé par un rapprochement entre les structures de différents systèmes langagiers (pour des définitions plus précises, voir les autres contributions à ce numéro). Celui-ci s'observe surtout dans les situations de contact extensif (Thomason \& Kaufman, 1988). Les conséquences de celle-ci peuvent amener à une simplification, où, comme nous tenterons de le démontrer ici à une complexification et une diversification des systèmes.

3 Le contact intense et ancien entre l'arabe maghrébin et le berbère s'inscrit parfois dans des dynamiques de convergence, lesquelles varient beaucoup en intensité d'une zone de contact à une autre (Kossmann, 2013). Les différences observables entre les situations de contact arabe-berbère nous apportent de précieuses données sur la diversité des conditions sociohistoriques de celles-ci (Souag, 2009). 
Dans les Babors, ce contact a conduit à l'arabisation d'une large part du massif, contre le maintien du berbère sur une zone s'étalant sur moins d'un tiers des Babors. L'introduction ancienne de l'arabe dans cette région qui n'a pas, contrairement aux autres massifs de Kabylie orientale, perdu sa langue berbère, est à l'origine d'un degré élevé de convergence entre les deux systèmes. Nous nous intéresserons ici aux conséquences de cette dernière sur le berbère tasahlit² ${ }^{2}$.

5 Les deux langues au centre de cette étude appartiennent au phylum afro-asiatique. Or nous savons que l'étude des phénomènes de convergence est particulièrement complexe en présence de langues apparentées génétiquement (Aikhenvald, 2007). La difficulté étant de différencier les emprunts ou phénomènes de contact des cognats et des tendances héritées. Aussi, il importe de procéder à des descriptions fines des phénomènes avant de tenter d'en affirmer la directionnalité avec prudence.

6 La négation est un domaine pour lequel l'arabe maghrébin et le berbère ont été particulièrement étudiés (Galand, 1994 ; Chaker \& Caubet 1996 ; Mettouchi, 1996, 2009 ; Lucas, 2007, 2013 ; Lafkioui \& Brugnatelli, 2017, 2020). Les phénomènes de convergence dans le domaine de la négation sont attestés dans les langues du monde (Willis, Lucas \& Breitbarth, 2013). Ce type de convergence a également fait l'objet d'études dans le contexte du contact arabe-berbère. Parmi les grandes problématiques soulevées par celle-ci figure la présence ou non parmi les différentes variétés de berbère et d'arabe maghrébin d'un négateur postverbal procédant de la grammaticalisation (plus ou moins importante) du pronom indéfini "chose» (Brugnatelli, 2014; Lafkioui \& Brugnatelli, 2020 ; Kahlouche, 2000 ; Mettouchi, 2012 ; Kossmann, 2013 ; Lucas, 2007, 2009, 2013, 2017; Souag, 2018). La grammaticalisation de "chose» en négateur postverbal est attestée en berbère tasahlit, à côté de celle d'autres éléments que nous listerons et dont nous chercherons les étymons. Fait plus inédit, les parlers tasahlit ont également grammaticalisé des verbes d'étymons arabes en négateurs verbaux, lesquels obéissent à une syntaxe différente de celle des négateurs d'étymon berbère ${ }^{3}$.

7 Nous chercherons dans le présent travail à décrire les « néo-négateurs ", leurs origines à travers leurs voies de grammaticalisation, ainsi que les changements que ceux-ci apportent sur la morphosyntaxe de la négation en berbère tasahlit. Cette analyse sera conduite en posant la question de la convergence entre le berbère septentrional et l'arabe maghrébin.

\section{Méthodologie}

8 L'aire $\mathrm{du}$ berbère tasahlit $(\approx 200000$ locuteurs $)$ est marquée par une variation diatopique très élevée, et comprend près d'une quinzaine de parlers correspondants à autant de confédérations distinctes, pour la plupart non décrits. Nous avons donc procédé à une enquête de terrain concernant la négation en tasahlit en mars 2020, venue renforcer des données prélevées durant plusieurs enquêtes de terrain depuis 2018. Durant cette enquête nous avons procédé à des entretiens enregistrés auprès de locuteurs originaires des 9 confédérations sahilophones suivantes: Aït Bouysef et Aït Laâlam (Tamridjet), Aït Felkaï (Darguina), Aït Segoual (Melbou et Ziama-Mansouriah), Aït Mhend (Aokas), Aït Hassaïn (Souk El Ténine), Aït Meraï (Kherrata), Aït Smail et Aït Mella (Oued El Bared). 
9 Ces données ont par la suite été comparées à celles d'autres langues berbères, de l'arabe maghrébin, de l'arabe levantin et de l'arabe classique.

Figure 1. Situation géographique de la Kabylie des Babors

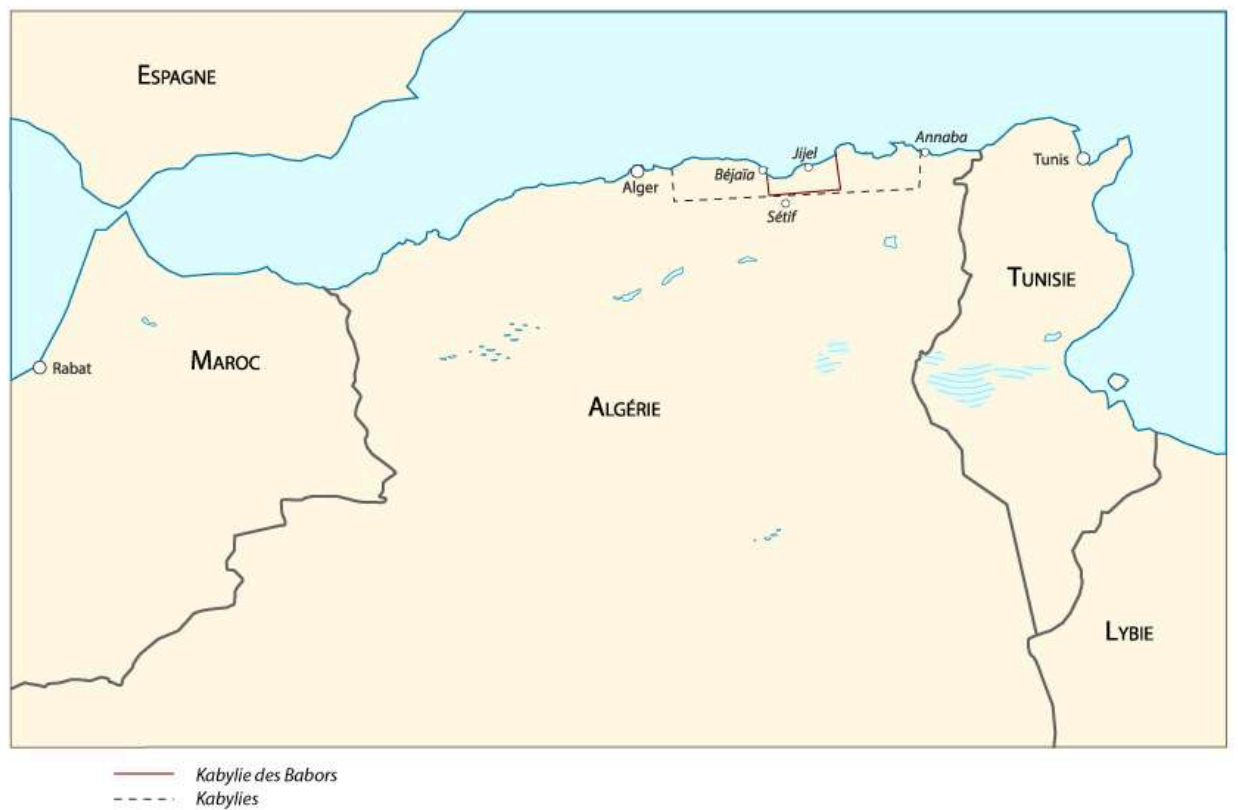

Figure 2. Aire de la Tasahlit

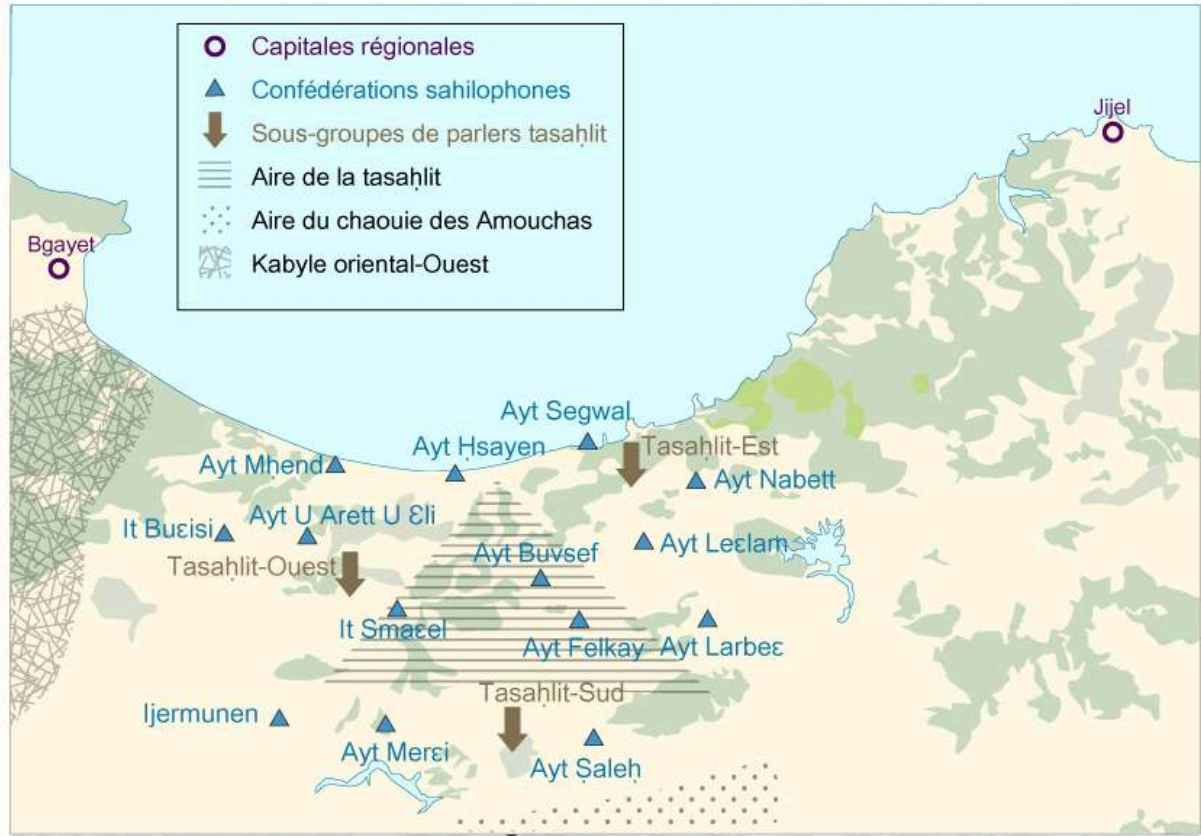




\section{La négation verbale en berbère}

10 Parmi les langues berbères, la négation non-emphatique en énoncé déclaratif d'un prédicat verbal est obtenue au moyen d'un ou de la combinaison des marquages suivants :

11 - Un négateur préverbal

12 - Un négateur postverbal

13 - Des conjugaisons spécifiques (dites négatives)

14 Le marquage par des conjugaisons négatives sera directement décrit dans la partie dédiée (sous-partie 5.4). Le seul marqueur tout à fait pan-berbère parmi ces trois éléments est le négateur préverbal (Galand 1994). Ce négateur est en effet représenté dans chacun des quatre grands blocs de parlers berbères (est, ouest, nord, sud). Il est également représenté dans toutes les variétés de berbère contemporaines (à l'exception du berbère égyptien, Souag 2009) par une forme commune, toujours composée d'un négateur représenté par la radicale $W$, souvent associée à un second élément $(A) R / L^{4}$.

15 Parmi les blocs septentrionaux et occidentaux, un nombre important de langues berbères possèdent également un marqueur de négation postverbal, dont l'usage présente différents degrés de grammaticalisation selon les parlers. Contrairement au négateur préverbal WR/L les étymons (sème + origine) de ces négateurs sont plus divers bien que le développement à partir d'éléments adverbiaux ou nominaux signifiants "chose » soit parmi les plus courants (Chaker, 1996; Kossmann, 2017; Lafkioui \& Brugnatelli, 2017, 2020). L'origine du développement d'un négateur postverbal, ainsi que la voie ayant amené le plus souvent à la grammaticalisation du pronom indéfini « chose » dans ce marqueur, est sujette à d'importants débats notamment sur le plan de convergences ou d'emprunts possibles avec l'arabe maghrébin (Chaker \& Caubet, 1996 ; Mettouchi, 1996 ; Rabhi 1996 ; Souag 2009 ; Kossmann, 2013 ; Lafkioui 2013 ; Brugnatelli, 2014 ; Lucas, 2007, 2009, 2013, 2017; Lafkioui \& Brugnatelli, 2020). Nous ne nous inscrirons pas ici dans ces problématiques, celles-ci exigeant une vision des données berbères et arabes dans leur ensemble.

16 Signalons enfin que, malgré l'apparente facilité à pointer un modèle unique de négation verbale en énoncé déclaratif, des travaux récents (Mettouchi, 1995, 1998, 2009) montrent que les conditions sémantico-pragmatiques de présence/absence de l'élément post verbal (et sa variation formelle) pointent vers une utilisation beaucoup plus subtile et très peu grammaticalisée des différentes particules (Mettouchi, communication personelle). Les différents marqueurs signalés plus hauts, pourraient donc selon les conditions de leur présence dans les différentes variétés, renvoyer historiquement ou en synchronie à des types sémantico-pragmatiques variés.

\section{Les marques de la négation verbale en berbère tasahlit}


Figure 3. Particule de négation pré-verbale dans les Babors berbérophones

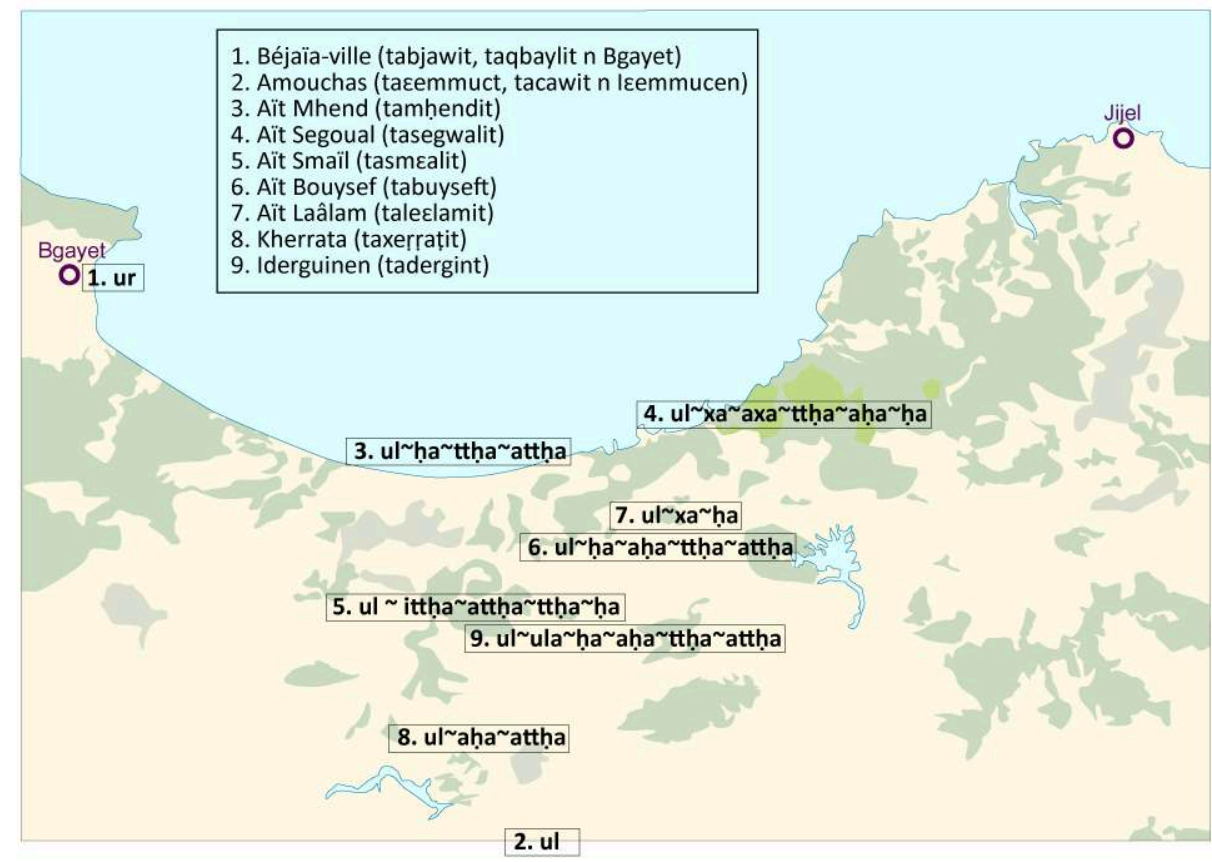

Figure 4. Particule de négation post-verbale dans les Babors berbérophones (les formes minoritaires localement sont données entre parenthèses)

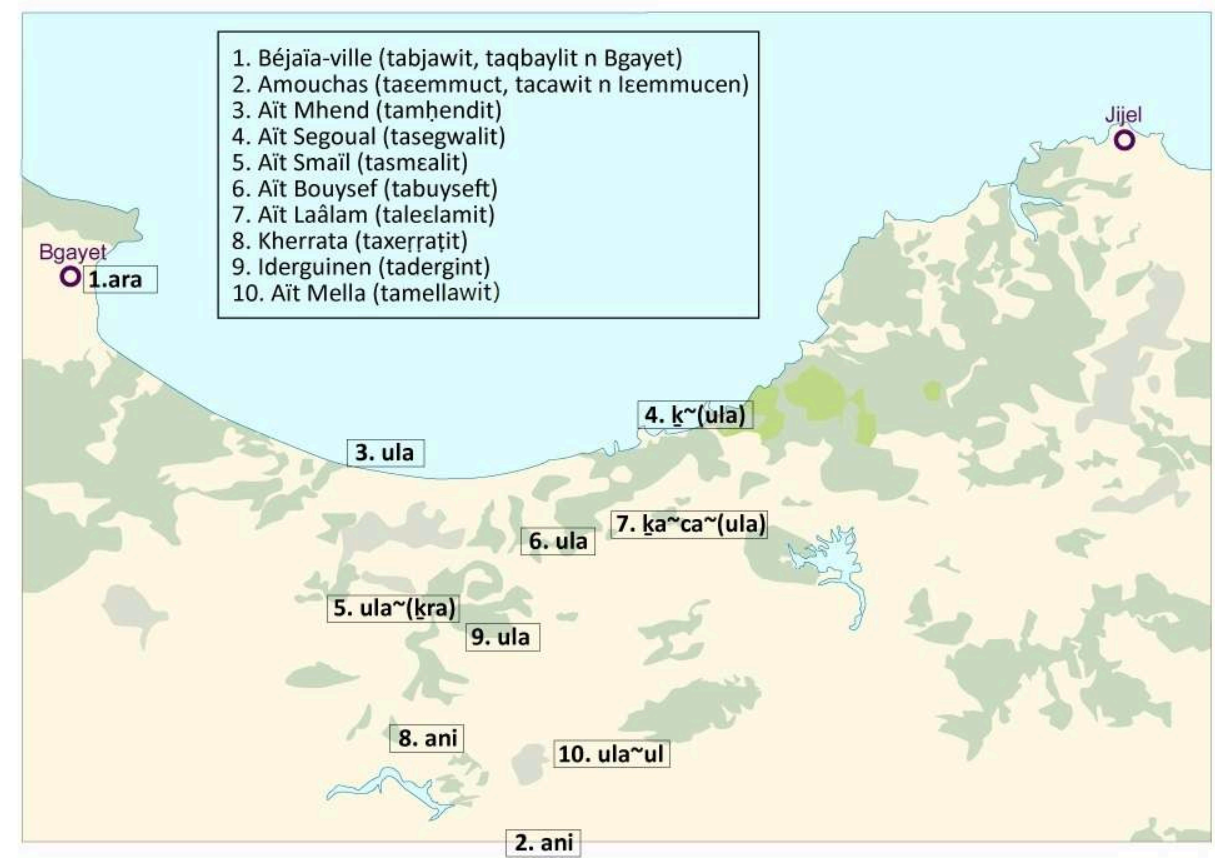

Le berbère tasahlit est concerné par les trois types de marquages présentés plus haut. Le premier d'entre eux, la particule W (+ AR/L) est représentée par la forme pantasahlit $\mathbf{u} \mathbf{l}^{5}$ (et sa variante conditionnée phonétiquement $\mathbf{u}$ ), et les néo-négateurs que nous décrirons plus bas. En berbère tasahlit, la négation verbale portée uniquement par l'élément préverbal est conditionnée par des environnements à forte contrainte 
syntaxique $e^{6}$, ainsi que par des registres littéraires particuliers (poésie, proverbes, etc.). Dans les parlers tasahlit qui ne connaissent pas de conjugaison négative, la négation préverbale constitue parfois l'unique marque de négation d'un énoncé verbal :

\begin{tabular}{|l|l|l|}
\hline \multicolumn{2}{|l|}{ Ex. 1 Aït Laâlam (2020) } \\
\hline a-xennic=adinat & ul & i-cca \\
\hline EL.M.SG-enfant=DEM.PROX & NEG1 & 3SG.M-manger $\backslash A C C$ \\
\hline «Ce garçon n'a pas mangé ! » \\
\hline
\end{tabular}

\begin{tabular}{|c|c|c|c|c|}
\hline tinnit & $n$ & ahentay $=a$ & $\mathbf{u}$ & $n t=t$ thibbi- $-\gamma$ \\
\hline DEM.ANAPH & GEN & PRES.3PL.F=DEIX.PROX & NEG & 3PL.F $=$ aimer $\backslash$ INT-1SG \\
\hline
\end{tabular}

18 Toutefois, dans la négation verbale canonique, le négateur préverbal est le plus souvent attesté avec des négateurs postverbaux. En tasahlit, les formes et les étymons de ces négateurs varient d'une confédération à une autre, voire au sein des diverses fractions d'une même confédération.

La forme la plus largement attestée est le NEG2 ula. Ce négateur est sans doute lié à l'adverbe pan-tasahlit ula "même, aussi » ( u uma ulamma etc.) assez largement attesté dans d'autres variétés de berbère-Nord (kabyle, atlas blidéen, chaoui etc.). Il n'est attesté ailleurs en berbère comme négateur postverbal que dans le parler sanhadja des Ayt Hmed (Rif central ${ }^{7}$ ).

\begin{tabular}{|c|c|c|c|c|}
\hline \multicolumn{5}{|c|}{ Ex. 3 Aït Bouysef (2020) } \\
\hline $\mathrm{ul}$ & ttrug-ex & ula & $g$ & $\underline{t} \mathrm{a}=\mathrm{ll}$ as $=\mathrm{t}$ \\
\hline NEG1 & sortir \INT-1SG & NEG2 & dans & SG.F $=$ obscurité $=\mathrm{SG} . \mathrm{F}$ \\
\hline
\end{tabular}

Dans le parler des Aït Felkaï, ula fonctionne également comme un négateur préverbal non suivi d'un second négateur postverbal ${ }^{8}$ :

\begin{tabular}{|l|l|l|l|}
\hline \multicolumn{2}{|l|}{ Ex. 4 Aït Felkaï (2020) } \\
\hline a=zgacbun & ula & i-ttwațțfay & sy=ahatayad \\
\hline EL.SG.M=rouge gorge & NEG & 3SG.M-saisir\INT & par=PROX \\
\hline
\end{tabular}


« On ne capture pas de rouge-gorge par ici »

D'après Kahlouche (2000) et Brugnatelli (2007: 404), cet adverbe, et par extension le NEG2 dans lequel il se serait grammaticalisé, correspondent à l'agglutination du négateur absolu $u$ avec une forme figée du verbe "être" la. Une construction en tasahlit pourrait renforcer cette hypothèse : des énoncés verbaux négatif absolus, dans lesquels le verbe est directement suivi de la sentence de négative figée ul illa «il n'y a pas ».

\begin{tabular}{|l|l|l|l|}
\hline \multicolumn{4}{|l|}{ Ex. 5 Aït Bouysef (2020) } \\
\hline ul & sagg-eX & ul & i-lla \\
\hline NEG1 & regarder \ACC-1SG & NEG1 & 3SG.M-exister \ACC \\
\hline \multicolumn{4}{|l|}{ «Je n'ai absolument pas regardé » } \\
\hline
\end{tabular}

Si cette proposition étymologique s'avère correcte, ce développement correspondrait au cycle de Croft (1991: 1-27). Cet auteur a proposé que les existentiels négatifs pouvaient être une source du marquage de la négation à travers un cycle de grammaticalisation à trois niveaux. Le premier niveau dépend de l'utilisation d'existentiels négatifs dans des constructions spécifiques; le second, de l'utilisation d'existentiels négatifs comme négateurs emphatiques (cf. exemple 5) et le troisième, de la réinterprétation des existentiels négatifs en tant que marqueurs généraux de la négation ( $c f$. exemples $3 \& 4$ et si l'étymologie proposée pour ula s'avère correcte).

Par ailleurs, on trouverait à deux reprises le négateur pan-berbère $\mathrm{W}$ dans une phrase verbale négative dotée de NEG1 ul et de NEG2 ula.

Une seconde forme - $u l$ - est attestée seulement comme variante facultative de ula. Ce NEG2 tout à fait identique au NEG1 pan-tasahlit ul correspond soit à une évolution de ula par érosion phonétique (chute du [a] final), soit à une répétition postverbale du NEG1 ul. Le second scénario est renforcé par le fait que la stratégie d'élaboration d'un NEG2 à partir de la forme de NEG1; une stratégie bien connue ailleurs, par exemple en portugais brésilien (Schwenter, 2005).

\begin{tabular}{|l|l|l|l|}
\hline \multicolumn{3}{|l|}{ Ex. 6 Aït Mella (2019) } \\
\hline ți-meččuk-in & ul & ggur-ent & ul \\
\hline EL.PL.F-filles-PL.F & NEG1 & partir $\backslash$ ACC-3PL.F & NEG2 \\
\hline « Les filles ne sont pas parties » \\
\hline
\end{tabular}

D'autres parlers tasahlit emploient également un négateur post-verbal ani, dont ils partagent l'usage avec quelques parlers kabyles orientaux-Est (Aït Amrous, Aït Melloul, etc.) de même qu'avec l'îlot chaouiphone septentrional des Amouchas? . 


Ex. 7 Aït Meraï (2019)
\begin{tabular}{|l|l|l|l|l|l|}
\hline ul & i-ssin & ani & menhu & d & netta \\
\hline NEG1 & 3 3SG.M-savoir \ACCNEG & NEG2 & qui & FOC & 35 SS.M \\
\hline
\end{tabular}
«Il ne sait pas de qui il s'agit »

Ce NEG2 pourrait correspondre à une grammaticalisation de l'interrogatif locatif ani « où » (Rabhi 1994). L'énoncé suivant (dans un parler préférant le NEG2 ula), actualise l'emploi de ce locatif à la suite d'une forme verbale négative. Ce type syntaxique et sémantique ${ }^{10}$ pourrait être à l'origine de la grammaticalisation du locatif ani en négateur verbal, bien que les raisons ayant amené à sa généralisation restent mystérieuses :

\begin{tabular}{|c|c|c|c|c|}
\hline \multicolumn{5}{|c|}{ Ex. 8 Aït Bouysef (2020) } \\
\hline ul & isca & ani & di & ${ }^{\mathrm{i}-}$ ssens \\
\hline NEG1 & 3SG.M-posséder $\backslash$ ACC & où & AOR & 3sG.M-passer la nuit $\backslash \mathrm{ACC}$ \\
\hline
\end{tabular}

Les dernières formes attestées comme NEG2 dans les parlers tasahlit correspondent à un groupe dérivé du pronom indéfini $k r a$ « chose ${ }^{11} »: \underline{k} r a \sim \underline{k} a^{12} \sim(e) \underline{k}^{13}$. Cette forme est également attesté dans le parler des Aït Youssi d'Enjil (Moyen-Atlas, Galand $1988: 222$ ), sa variante palatalisée $c a$ est fréquente dans le Moyen-Atlas, en rifain ${ }^{14}$ ainsi qu'en chaoui. Selon nous, ces formes correspondent soit à des variantes phonétiques présentant différents degrés d'érosion phonétique, soit à des variantes historiques correspondant à des formes ultérieures à la formation du pronom indéfini $\mathrm{kra}^{15}$. Ces négateurs correspondant à une grammaticalisation du pronom indéfini «chose", comme la majorité des négateurs postverbaux attestés en nord-berbère, entrent dans les débats évoqués plus tôt.

28 A l'échelle du berbère, le degré de variation proposé par le négateur postverbal en berbère tasahlit est exceptionnel. Nous pensons que ce dernier trouve son origine à la fois dans un phénomène de contact intra-berbère - ce qui semble apparaître dans le cas du NEG2 ani également attesté en chaoui septentrional ainsi qu'en kabyle oriental - et de développement internes spéciaux à la région (cas de la grammaticalisation de ula).

Enfin, des conjugaisons négatives attestées dans l'ensemble berbère, le berbère tasahlit n'atteste que d'un accompli négatif, qui n'est lui-même relevé que dans une partie de cette aire. Nous reviendrons plus tard sur ce point précis, après avoir présenté les deux groupes de «néo-négateurs » au centre de notre attention. 


\section{Les négateurs issus de la grammaticalisation de thinu}

\begin{tabular}{|c|c|c|c|c|c|}
\hline \multicolumn{6}{|c|}{ Ex. 10 Aït Bouysef (2020) } \\
\hline $\mathrm{ta}=\mathrm{ha}$ & d & ta-yyin-t & attha & d & ti-sefsi-t \\
\hline DEM $\backslash S G . M=P R O X$ & COP & EL.SG.F-marmite-SG.F & NEG & COP & EL.SG.F-pot à lait-SG.F \\
\hline
\end{tabular}

Plusieurs variantes de cette première série de néo-négateurs ont été signalées par nos prédécesseurs en tasahlit et en kabyle : Rabdi (2004:110) dans le parler kabyle oriental des Ihbachen, Rabhi (1994) et Berkaï (2011:99) pour le parler tasahlit des Aït Mhend. Cette première série de néo-négateurs verbaux présente en effet un certain nombre de variantes correspondant à différents degrés d'érosion phonétique du verbe grammaticalisé tḥu :

a) itha > ittha (désemphatisation)

b) attha (harmonisation vocalique)

c) ttha aha > ha (différents degrés d'érosion phonétique)

Ces différentes formes correspondent en synchronie à des variantes géographiques : certains parlers n'en connaissant qu'une, tandis que d'autres en utilisent plusieurs à la fois ( $c f$. figures $3 \& 4$ ). Les usages de ces négateurs peuvent varier d'un parler à un autre : si leur utilisation dans le cadre de la négation verbale canonique est celle que nous avons le plus largement observée durant notre enquête, des usages secondaires sont également parfois attestés. Dans les parlers qui actualisent des usages secondaires, ces derniers peuvent se voir spécialisés à côté d'une forme correspondant à un degré d'érosion phonétique, contre un autre usage associé à un autre degré d'érosion.

Chez les Aït Meraï (Kherrata) nous avons par exemple relevé que la forme aḥa était spécialisée dans l'infirmation d'une phrase verbale conjuguée à l'aoriste l'intensif, tandis que attha était réservée exclusivement à la négation de l'aoriste. Dans le parler kabyle oriental des Ihbachen, Rabdi (2004) précise que l'usage de itha était à la fois archaïque (récolté uniquement auprès de femmes âgées) et expressif. Chez les Aït Mhend, nous avons remarqué que la forme attha était également souvent utilisée dans le cadre de la négation de prédicats non-verbaux :

\begin{tabular}{|c|c|c|}
\hline attḥa & d & leali-n \\
\hline NEG & $\mathrm{COP}$ & bon-PL $\backslash \mathrm{M}$ \\
\hline
\end{tabular}

Chez les Aït Bouysef, attha est utilisé dans la négation nominale :

TIPA. Travaux interdisciplinaires sur la parole et le langage, 37 | 2021 


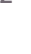

D'après nos observations empiriques sur le terrain au printemps 2020, les variantes de ce néo-négateur nous ont paru d'un usage particulièrement fréquent dans les discours des jeunes locuteurs. Nos observations auprès d'enfants appartenant à la confédération de Aït Hassaïn dans le village de Lota (commune de Souk El Ténine) tendraient même à indiquer l'éviction en cours de ul par ces néo-négateurs. Comparativement, nous avons récolté en 2015 un corpus de contes auprès de locutrices de différents parlers de la région, souvent âgées et monolingues, dans lequel l'usage des néo-négateurs est peu courant et systématiquement plus rare que celui exercé par leurs équivalents en ul $(+$ NEG2). Il conviendrait bien sûr de vérifier ces données empiriques au moyen de statistiques, réalisés sur des corpus langagiers variées prenant notamment en compte la variation intergénérationnelle et diatopique. Ces résultats pour l'aire tasahlit vont dans le sens inverse de ceux du kabyle des Ihbachen, pour lequels Rabdi (2004) signale l'absence totale de la variante locale de ces néo-négateurs dans les parlers des jeunes : une évolution qui pourrait s'expliquer par des phénomènes de koinéisations régionales dans les parlers des centres urbains ou semi-urbains régionaux (kabyle de Béjaïa pour le kabyle oriental / parlers de Kherrata, de Souk El Ténine et d'Aokas pour la tasahlit).

Signalons enfin que plusieurs informateurs locuteurs de kabyle extrême-occidental (kabyle des piémonts du Djurdjura occidental) nous ont indiqué que leurs parlers ou ceux de leur voisinage connaissaient une négation à un seul morphème préverbal ha. Ne disposant d'aucune information complémentaire sur la répartition de ce négateur, de même que sur son usage précis, nous espérons que des enquêtes ou des travaux sur la négation dans cette région ${ }^{21}$ viendront renforcer nos connaissances. Si l'usage de négateurs issus de la grammaticalisation de tḥu était également avéré dans cette région, des hypothèses supplémentaires pourraient être faites sur son origine et sa diffusion.

TIPA. Travaux interdisciplinaires sur la parole et le langage, 37 | 2021 


\section{Les négateurs issus de la grammaticalisation de $x t ̦ u$} constitué que de deux variantes : $x a$ et $a x a$. Contrairement aux formes présentées plus tôt, celles-ci ne sont rencontrées que dans une zone très réduite de l'aire tasahlit, partout en concurrence avec les formes du premier groupe. Les parlers concernés sont les variétés dites tasahlit orientales, groupe de parler assez individualisés (Garaoun 2019) pratiqués à la frontière de l'arabe jijélien par des populations très largement bilingues berbère tasahlit et arabe jijélien. Dans ces parlers, tantôt les deux formes relevées sont attestées toutes deux comme variantes (Aït Laâlam), tantôt la forme $x a$ est seule attestée (Aït Segoual). Ailleurs en berbère, une forme phonétiquement proche partageant possiblement le même étymon que ces néo-négateurs - $x u$ - est attestée en tamazight du Moyen-Atlas, dans le parler des Ait Wirra (Oussikoum 2013:92).

En synchronie, les parlers tasahlit présentent plusieurs éléments provenant de la racine arabe XT «manquer, faire défaut»: les adverbes négatifs emphatiques xat (Tasahlit-Est) et xați (reste de l'aire tasahlit ${ }^{22}$ ), qui fonctionnent également dans plusieurs parlers suivis de la copule $d$ comme des négateurs de phrases nominales.

\begin{tabular}{|l|l|l|}
\multicolumn{3}{|c|}{ Ex. 10 Aït Bouysef (2020) } \\
\hline xați & d & cmina \\
\hline NEG & PRED & 2SG.F \\
\hline \multicolumn{3}{|l}{ "Ce n'est pas toi » } \\
\hline
\end{tabular}

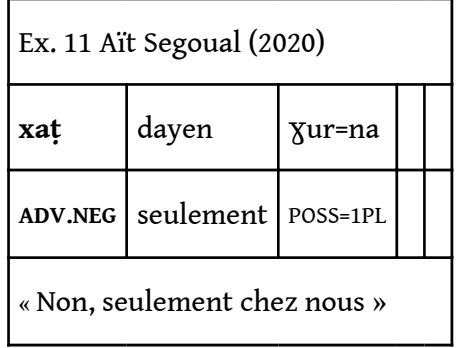

Le verbe $x t ̦ u$ (plus au moins figé dans certains parlers à la 3sG ixța (m) / texța (f) «il/elle est différent/e, il/elle est exclu » (Berkaï, 2014 : 1045). Cette dernière forme verbale est connue dans toute l'aire tasahlit, son caractère figé à la 3sG dans certains parlers de cette région indique le processus en cours de grammaticalisation de ce verbe.

\begin{tabular}{|l|l|}
\hline \multicolumn{2}{|l|}{ Ex. 12 Aït Laâlam (2019) } \\
\hline wa=nahu & i-xṭa \\
\hline DEM.M=DIST.SG & 3SG.M-être exclu $\backslash$ ACC \\
\hline « celui-ci il est exclu » \\
\hline
\end{tabular}



XT verbe ou négateur n'est guère surprenant: cette racine est liée à la notion sémantique d'exclusion, et est à l'origine de négateurs en berbère comme dans la langue dont elle est originaire. Ajoutons également que contrairement à la série issue de thhu, les néo-négateurs $x a$ et axa sont d'un usage limité à quelques parlers, dans lesquels ils sont en compétition avec les dérivés du premier groupe. Les parlers tasahlit orientaux étant en situation de contact avec les parlers tasahlit occidentaux, spécialement dans les quartiers en cours d'urbanisation de Letnayen, Tizi L'Oued et Tizi War (commune de Souk El Ténine), dans lesquels beaucoup de familles originaires des confédérations tasahlit orientales se sont exilées durant les dernières décennies. Ici encore, une étude statistique à partir d'un corpus permettrait de mieux observer cette compétition entre formes et les principales tendances résultant de ces contacts intralinguistiques.

\section{Les particularités morphosyntaxiques des néo- négateurs}

La négation canonique verbale au moyen des deux séries de néo-négateurs décrites fonctionne différemment des séries réalisées à partir du négateur pan-berbère ul. Certains des comportements spécifiques à la négation à partir des néo-négateurs allant jusqu'à éloigner la morphosyntaxe de la négation des parlers tasahlit de ses modèles les plus répandus en berbère. Sans prétendre clarifier ici le fonctionnement historique de la négation en tasahlit, nous essayerons néanmoins de mettre en exergue ce qui, dans les parlers étudiés, semble en cours de déviation par rapport à des schémas largement partagés par les variétés contemporaines et pourrait correspondre soit à des développements internes soit à des convergences syntaxiques avec l'arabe.

TIPA. Travaux interdisciplinaires sur la parole et le langage, 37 | 2021 


\subsection{L'érosion de l'accompli négatif} est toujours facultatif dans les énoncés verbaux négatifs marqués par les néonégateurs ${ }^{27}$ :

\begin{tabular}{|l|l|}
\hline \multicolumn{2}{|l|}{ Ex. 13 Aït Mhend (2020) } \\
\hline attḥa & ibzeg \\
\hline NEG & 3SG.M.être humide $\backslash$ ACC \\
\hline «Il n'est pas humide » \\
\hline
\end{tabular}

\begin{tabular}{|l|l|}
\hline \multicolumn{2}{|l|}{ Ex. 14 Aït Mhend (2020) } \\
\hline atthạa & ibzig \\
\hline NEG & 3SG.M.être humide \ACC.NEG \\
\hline « Il n'est pas humide » \\
\hline
\end{tabular}

La perte du caractère obligatoire de ce marquage s'explique sans doute par le fait que les néo-négateurs soient arrivés dans un système qui tendait déjà à perdre ses marquages aspectuels spécifiques pour ne plus marquer la négation qu'au niveau du négateur lui-même. Dans la négation verbale modale/non-déclarative ce même caractère facultatif (et parfois accompagné d'une valeur pragmatique dans le discours) du marquage de l'accompli négatif est observé.

\subsection{L'érosion de l'attraction des satellites du verbe}

Une des particularités syntaxiques des langues berbères est un phénomène complexe d'attraction des pronoms clitiques et déictiques devant le verbe dans certaines situations syntaxiques: parmi celles-ci la négation, mais aussi selon les variétés la focalisation, l'interrogation. En dehors de ces contextes d'attraction, l'ordre 
d'apparition des clitiques le plus communément admis en berbère devant le verbe est de type : «classiques » et présentent des «déviances" dans le fonctionnement de ces attractions. C'est le cas de beaucoup de parlers tasahlit, qui ne respectent pour la plupart ni l'ordre classique en situation non attirée, ni celui correspondant à une situation d'attraction : on observe dès lors des constructions " déviantes " parfois très complexes variant d'un parler à un autre et selon les associations de satellites verbaux. C'est le cas par exemples chez les Aït Bouysef :

\begin{tabular}{|l|}
\hline Ex. 15 Aït Bouysef (2020) \\
\hline i-bbi=yay=dd=i \\
\hline 3SG.M-apporter $\backslash A C C=O I 1 S G=V E N T=O D 3 S G . F$ \\
\hline « Il me l'a apporté » \\
\hline Verbe + Objet indirect + Déictique d'orientation verbale + Objet direct \\
\hline
\end{tabular}

\begin{tabular}{|l|l|l|}
\hline \multicolumn{2}{|l|}{ Ex. 16 Aït Bouysef (2020) } \\
\hline u & s=ne-kffa=yadd=ut & ula \\
\hline NEG1 & OI3SG.M=1PL-donner $\backslash$ ACC=VENT=OD3SG.M & NEG2 \\
\hline « Nous ne le lui avons pas donné » \\
\hline \multicolumn{2}{|l|}{ Objet indirect + Verbe + Déictique d'orientation verbale + Objet direct } \\
\hline
\end{tabular}

64 Le stade maximal de la «déviance » dans l'application de l'attraction des satellites verbaux est représenté par le perte totale de cette dernière. Celle-ci n'est attestée à notre connaissance qu'en berbère oriental, dans les oasis d'Awjila (Van Putten \& Souag 2015 : 42) et de Sokna (Souag 2017) en Lybie, et de Siwa en Égypte (Schiattarella 2015 : 105).

En berbère tasahlit, nous pouvons néanmoins constater une perte complète de l'attraction dans certains contextes syntaxiques. La négation est attestée dans la totalité des parlers tasahlit uniquement dans les serments négatifs (réalisés au moyen du négateur ma) et les négations emphatiques (réalisées au moyen de *maci), ainsi que dans les sentences négatives formulées au moyen des néo-négateurs.

Ex. 17 Aït Bouysef (2020) 


\begin{tabular}{|l|l|}
\hline ifa=yat & inili \\
\hline 3SG.M $\backslash A C C=3 S G . M$ & tout à l'heure (passé) \\
\hline « Il l'a trouvé tout à l'heure » \\
\hline
\end{tabular}

\begin{tabular}{|l|l|l|l|}
\hline \multicolumn{4}{|l|}{ Ex. 18 Aït Bouysef (2020) } \\
\hline $\mathbf{u}$ & t=ifa & ula & mați \\
\hline NEG1 & 3SG.M=3SG.M \ACC & NEG2 & du tout \\
\hline « Il ne l'a pas trouvé du tout » \\
\hline
\end{tabular}

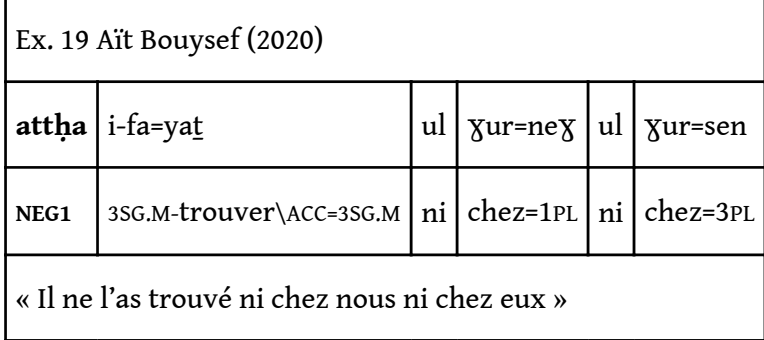

Comme l'érosion de l'accompli négatif, celle de l'attraction des satellites verbaux semble liée à des motivations internes d'affaiblissement : celle-ci est en effet attestée à différents niveaux un peu partout en nord-berbère. De manière similaire à l'érosion de l'accompli négatif, cette loi syntaxique pan-berbère n'est maintenue que dans les énoncés verbaux déclaratifs employant la particule pan-berbère ul, tandis qu'elle disparaît complètement avec les néo-négateurs ainsi que dans les énoncés négatifs nondéclaratifs réalisés à partir de négateurs d'étymons arabes.

\subsection{La négation de l'aoriste + particule}

Dans beaucoup de variétés de berbères-Nord, la négation de l'aoriste n'est possible qu'au moyen de l'aoriste intensif sans particule d'aoriste; dans certaines variétés, un thème d'inaccompli négatif est observé dans ce contexte. L'origine de ces développements est largement débattue, bien que ceux-ci soient souvent présentés comme des développements plutôt que des fonctionnements anciens (Galand $1994: 180$, Chaker, 1996, Mettouchi, 2009).

En tasahlit, le négateur préverbal ul ne s'observe que dans la flexion négative d'énoncés d'aoriste intensif sans particule d'aoriste ${ }^{28}$; tandis qu'avec les néo-négateurs, il est possible de fléchir négativement tous les types d'énoncés aoristes, avec particule ou sans, à l'aoriste comme à l'aoriste intensif :

69 Aït Bouysef $a w i$ « emporter, apporter » à la $3^{\text {ème }}$ personne du singulier masculin

\begin{tabular}{|l|l|l|}
\hline & Enoncé positif & Enoncé négatif \\
\hline
\end{tabular}




\begin{tabular}{|l|l|l|}
\hline Aoriste $+d i:$ & di ${ }^{\mathrm{i}} \mathrm{wi}$ & $\mathbf{( a ) ( t t ) h a ~ d i ~}{ }^{\mathrm{i}} \mathrm{wi}$ \\
\hline Aoriste intensif : & ittawi & $\mathbf{u l ~ i t t a w i ~} \sim(\mathbf{a})(\mathbf{t t})$ ḥa ittawi \\
\hline Aoriste intensif $+d i:$ & di ittawi & $\mathbf{( a ) ( t t ) h ̣ a ~ d i ~ i t t a w i ~}$ \\
\hline
\end{tabular}

70 En négation verbale modale/non-déclarative (ex. 20: négation prohibitive, ex. 21 : négation d'une proposition) on rencontre également cette possibilité d'associer un négateur à un thème d'aoriste ou d'aoriste intensif avec particule. Ici aussi, le comportement des néo-négateurs et des négateurs de négation verbale modale/nondéclarative vient s'opposer à celui du négateur ul.

\begin{tabular}{|l|l|l|l|l|}
\hline \multicolumn{5}{|l|}{ Ex. 20 Aït Segoual (2020) } \\
\hline ahu & da & ddu-t & i & lahen \\
\hline OPT.NEG $^{29}$ & AOR & aller INACC-2SG & vers & là-bas \\
\hline \multicolumn{5}{|l}{} \\
« Ne va pas là-bas » \\
\hline
\end{tabular}

\begin{tabular}{|l|l|l|}
\hline \multicolumn{2}{|l|}{ Ex. 21 Aït Hassaïn (2019) } \\
\hline maci & di & ggd-et \\
\hline NEG.EMPH $^{30}$ & AOR & avoir peur \INACC-2SG \\
\hline «Ce n'est pas que tu auras peur » \\
\hline
\end{tabular}

\subsection{L'absence de négateur post verbal}

71 La dernière caractéristique des néo-négateurs préverbaux concerne leur grammaticalisation comme négateurs fonctionnant seuls, généralement non suivis d'un négateur postverbal ${ }^{31}$. Les néo-négateurs, contrairement à ul, ne semblent donc pas s'inscrire dans le cycle de la négation décrit par Jespersen (1917). En effet, le modèle de négation verbale déclarative pan-tasahlit présente un modèle de négation à deux négateurs - préverbal et postverbal - dans lequel le premier élément correspond à l'élément originel, et l'élément postverbal à un rajout en cours de grammaticalisation venant appuyer le NEG1. Selon le cycle de la négation proposé par Jespersen, on pourrait s'attendre à un renforcement de la grammaticalisation des NEG2, laquelle pourraient précéder une chute du négateur original (NEG1). Cette situation est d'ores et déjà attestée dans quelques parlers berbères (Lafkioui \& Brugnatelli, 2020). L'inexistence d'un NEG2 grammaticalisé dans les phrases négatives comportant un néo-négateur préverbal indique la non-activation du cycle de Jespersen auprès de ces formes, et donc son non-transfert à partir de la négation originelle vers ces modèles de néo-négation en tasahlit. Il est intéressant de constater que ces néo-négateurs d'étymon arabe ne se comportent également pas sur ce point de manière convergente avec l'arabe 
maghrébin ${ }^{32}$ qui a complètement grammaticalisé son négateur postverbal dans la négation verbale déclarative.

Cette syntaxe est aussi celle des négateurs de négation verbale modale/non-déclarative empruntés à l'arabe *maci ${ }^{33}$ et xaț (i) (négation emphatique, $c f$. ex. 22) et ma (négation de serments). En effet, ces derniers correspondent à des négateurs toujours préverbaux pour lesquels la présence d'un négateur ou d'un complémenteur postverbal est exclue $^{34}$.

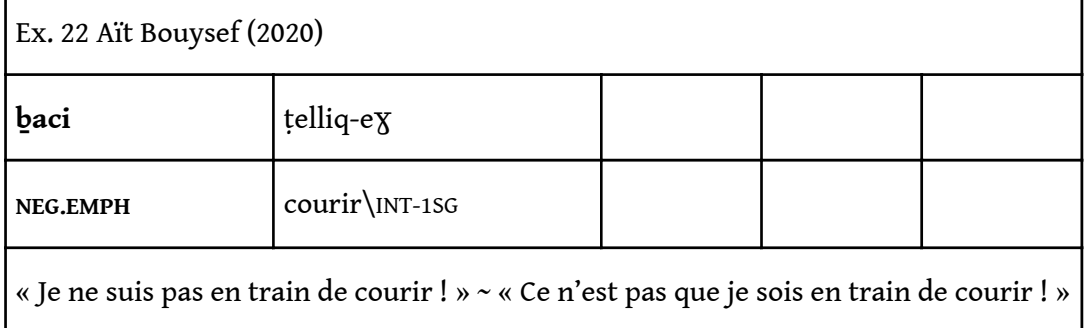

\begin{tabular}{|c|c|c|c|c|c|}
\hline \multicolumn{6}{|c|}{ Ex. 23 Aït Hassaïn (2019) } \\
\hline f & $i=x e f$ & $n$ & baba & ma & Ealim-ey \\
\hline sur & EL.M.SG=tête & GEN & père \1SG & NEG & connaître $\backslash A C C . N E G-1 S G$ \\
\hline
\end{tabular}

Les quatre particularités syntaxiques venant différencier l'usage des néo-négateurs de celui du négateur originel ul (+ NEG2) dans la négation verbale déclarative, ont permis de relever une ressemblance de comportements syntaxiques entre ces néo-négateurs et les négateurs de négation verbale modale/non-déclarative empruntés à l'arabe. arabe maghrébin, Chatar-Moumni (2008) présente le négateur māši comme un négateur verbal emphatique de l'arabe marocain. Ses contextes utilisation sont très similaires à celle que connaisse *maci et xaț(i) en tasahlit.

Concernant le négateur d'assertion $m a^{35}$, signalons qu'en arabe jijélien, la particularité des phrases verbales assertives négatives est de présenter une négation à un seul négateur (préverbal), contre une négation déclarative présentant un négateur postverbal $\check{s}(i)$ obligatoire ( $c f$. ex. 26 et \& 27). Ce comportement correspond soit à une influence soit à un conservatisme de la langue religieuse ${ }^{36}$ qui ne connaît pas de négateurs postverbaux (ex. 24).

Le fonctionnement de l'arabe jijélien est fortement parallèle à celui du berbère tasahlit, dans lequel ma emprunté fonctionne également toujours seul, sans négateur postverbal. Ce qui indique que l'emprunt de ma en tasahlit a impliqué un transfert de structures syntaxiques de l'arabe vers le berbère, à travers l'utilisation d'un négateur spécialisé dans le domaine assertif, fonctionnant sans négateur postverbal ${ }^{37}$.

\begin{tabular}{|l|l|}
\hline \multicolumn{2}{|l|}{ Ex. 24 arabe classique } \\
\hline $\mathbf{l a}$ & Pa-qūlu=laka \\
\hline
\end{tabular}




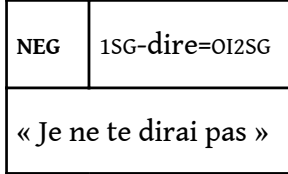

\begin{tabular}{|l|}
\hline Ex. 25 arabe jijélien (Jijel-ville, 2019) \\
\hline$n$-ḳull=lək \\
\hline 1SG-dire=OI2SG \\
\hline «Je te dirai »
\end{tabular}

\begin{tabular}{|l|l|l|}
\multicolumn{3}{|l|}{ Ex. 26 arabe jijélien (Jijel-ville, 2019) } \\
\hline ma & n-ḳūl=lək & š(i) \\
\hline NEG1 & 1SG-dire=OI2SG & NEG2 \\
\hline « Je ne te dirai pas » \\
\hline
\end{tabular}

\begin{tabular}{|l|l|l|}
\hline \multicolumn{3}{|l|}{ Ex. 27 arabe jijélien (Jijel-ville, 2019) } \\
\hline wəḷ̣āhi & ma & n-ḳūl=ək \\
\hline Par Dieu & NEG & 1SG-dire=OD2sg \\
\hline «Par Dieu je ne te dirai pas! » \\
\hline
\end{tabular}

77 De toute évidence, les lois syntaxiques des négateurs verbaux non déclaratifs empruntés *maci, xaț(i) et ma ont été partiellement transférés de l'arabe sur le berbère lors de leur emprunt. La proximité entre les lois régissant la négation au moyen de ces négateurs non déclaratifs et celle des néo-négateurs décrits plus tôt, vient confirmer l'hypothèse d'un usage d'origine non déclaratif de ces néo-négateurs.

78 Nous avons vu que les fonctionnements de ces négateurs empruntés connaissaient en arabe maghrébin une syntaxe très similaire, ce qui nous invite à postuler que leur emprunt s'est fait en même temps qu'un transfert d'une partie au moins de leur fonctionnement dans la langue d'origine. Des études plus approfondies sur le fonctionnement des différents négateurs de négation non-déclaratives en arabe maghrébin nous permettront sans doute de déterminer l'importance et les limites précises de ce transfert.

\section{Conclusion}

79 Nous avons présenté les comportements de deux séries de négateurs verbaux grammaticalisées à partir d'éléments empruntés à l'arabe. En berbère tasahlit, ces néonégateurs viennent, dans la négation verbale, provoquer ou renforcer plusieurs 
changements morphosyntaxiques. Ces transformations vers des modèles éloignés de ceux des parlers berbères conservateurs, et souvent similaires à ceux de l'arabe, méritent d'être questionnés sous l'angle de la convergence ${ }^{38}$, bien que souvent des motivations internes puissent également être invoquées. Nous avons décrit que l'usage des néo-négateurs entrainait : véritable fourmillement de négateurs verbaux attesté dans cette région. Il apparaît clairement que cette diversité, aujourd'hui menacée par les phénomènes de koinéisation régionale, révèle une histoire complexe: marquée aussi bien par un isolement géographique et communautaire que par d'importants contacts intra- et extra-berbères. On peut également se demander quel sera l'avenir d'une telle diversité de formes et quelle sera l'issue de la compétition en cours entre celles-ci, à l'heure de la progression galopante des koinès (semi-)urbaines. 


\section{BIBLIOGRAPHIE}

Berkaï, A. (2011) Les spécificités morphosyntaxiques du parler kabyle d'Aokas, Asinag, nº 6, Rabat: Institut Royal de la Culture Amazighe, p. 95-114.

Berkaï, A. (2014) Essai d'élaboration d'un dictionnaire tasahlit (parler d'Aokas)-français, Thèse doctorale, Tizi-Ouzou: Université de Tizi-Ouzou.

Brugnatelli, V. (2007) Problème de la négation en berbère: à propos de l'origine d'ulac, ula, ula d, in Proceedings of the 13th Italian Meeting of Afro-Asiatic Linguistics, Udine, p. 401-405.

Brugnatelli, V. (2011) Négations, participes et figement en berbère: nouvelles hypothèses, in A. Mettouchi (ed.), Parcours berbères. Mélanges offerts à Paulette Galand-Pernet et Lionel Galand pour leur 90e anniversaire, Köln: Köppe, p. 521-532.

Brugnatelli, V. (2014) Berber negation in diachrony, in The diachrony of negation, Amsterdam: John Benjamins, p. 167-184.

Chaker, S. \& D. Caubet (1996). La négation en berbère et en arabe maghrébin, 334, Paris: L'Harmattan.

Chaker, S. (1996) Quelques faits de grammaticalisation dans le système verbal berbère, Paris: Bulletin de la Société de Linguistique de Paris.

Chaker, S. (1997) Quelques faits de grammaticalisation dans le système verbal berbère, Mémoires de la Société de Linguistique de Paris, p. 103-121.

Chatar-Moumni, N. (2008) Quelques aspects du fonctionnement de la négation en arabe marocain, La linguistique, 44, 2, p. 81-98.

Croft, W. (1991) The evolution of negation, Journal of linguistics, 27.1, p. 1-27.

Dallet, J. M. (1982). Dictionnaire kabyle-français : parler des At Mangellat, Algérie, 2, Louvain: Peeters Publishers.

Delheure, J. (1984) Agraw n yiwalen tumzabt $t$-tfransist, Dictionnaire mozabite-français, Paris: SELAF/ Peeters.

El Arifi, S. (2016) Tamazight de l'Atlas Blidéen, Alger: Haut-Commissariat à l'Amazighité, Office des Publications Universitaires.

Galand, L. (1988) Le berbère (Dissertation Doctorale), Paris: Centre National de la Recherche Scientifique.

Galand, L. (1994) La négation en berbère, Matériaux arabes et sudarabiques, Paris: GELLAS, nouvelle série, 8, p. 169-181.

Galand, L. (2010) Regards sur le berbère, Milano: CSCS di Milano.

Garaoun, M. (2018) Noms à préfixes berbères en arabe jijélien. Comparaison entre le parler du centre-ville de Jijel et celui des Bni Mâad de Ziama-Mansouriah, Actes des 20èmes Rencontres Jeunes Chercheurs en Sciences du Langage.

Garaoun, M. (2019) Variation et classification en berbère-Nord : le cas des parlers tasahlit, Actes des 21èmes Rencontres Jeunes Chercheurs en Sciences du Langage.

Jespersen, O. (1917) Negation in English and Other Languages, Copenhagen: Host. 
Kahlouche, R. (2000) Le présentatif négatif ulac 'il n'y a pas', est-il de souche berbère ou un emprunt à l'arabe ?, Etudes berbères et chamito-sémitiques. Mélanges offerts à Karl-G. Prasse, Paris: Paris-Louvain (Peeters), p. 233-236.

Kossmann, M. (2013) The Arabic influence on northern Berber, Leiden: Brill.

Lafkioui, M. (2007) Atlas linguistique des variétés berbères du Rif, Cologne: Rüdiger Köppe Verlag.

Lafkioui, M. (2013) Reinventing negation patterns in Moroccan Arabic, in Lafkioui, M. (Ed.) African Arabic: Approaches to Dialectology, Berlin: De Gruyter, p. 51-94, (Trends in Linguistics. Studies and Monographs, 258).

Lafkioui, M. \& Brugnatelli, V. (2017) Negation in Berber: variation, typology and evolution, Studies in Language.

Lafkioui, M., \& Brugnatelli, V. (2020). Negation in Berber: variation, evolution, and typology, Linguistics.

Loubignac, V. (1924) Etude sur le dialecte berbère des Zaïan et Aït Sgougou: Textes et lexique II, Paris: Ernest Leroux.

Lucas, C. (2007) Jespersen's cycle in Arabic and Berber, Transactions of the Philological Society, 105, 3, p. 398-431

Lucas, C. (2009) The development of negation in Arabic and Afro-Asiatic, Cambridge: University of Cambridge.

Lucas, C. (2013) Negation in the history of Arabic and Afro-Asiatic, in Willis, D., Lucas, C. \& A. Breitbarth (eds) The development of negation in the languages of Europe and the Mediterranean, 1: Case studies, Oxford: Oxford University Press, p. 399-452.

Marçais, P. (1956) Le parler arabe de Djidjelli, Nord constantinois, Algérie, 16, Paris: Librairie d'Amérique et d'Orient.

Marcy, G. (1936) Note sur le pronom relatif-sujet et le pseudo-participe dans les parlers berbères, Bulletin de la Société Linguistique de Paris, 37, 1, p. 56-57

Mettouchi, A. (1996) Remarques sur la négation en berbère (kabyle), Mémoires de la Société de Linguistique de Paris, nouvelle série, 4, p. 105-116.

Mettouchi, A. (1998) Aspect et négation: remarques sur l'inaccompli et la négation en anglais et en berbère (kabyle), in Borillo, A., Vetters, C. \& M. Vuillaume (eds), Regards sur l'aspect, Amsterdam: Editions Rodopi, p. 191-205.

Mettouchi, A. (2005) Nonverbal and verbal negations in Kabyle (Berber), Studies in African Linguistic Typology, 64, p. 263.

Mettouchi, A. (2009) The system of negation in Berber, in Cyffer, N., Ebermann, E. \& G. Ziegelmeyer (eds), Patterns of Negation in West African Languages, Amsterdam: Benjamins, p. 287-306.

Mettouchi, A. (2012) Négation, Encyclopédie berbère, vol. 33, p. 5392-5399.

Naït-Zerrad, K. (2005) Procédés d'auxiliation et grammaticalisation en berbère, Faits de langues, $\mathrm{n}^{\circ}$ 26, Les langues chamito-sémitiques (afro-asiatiques), Vol. 1, p.241-246.

Oussikoum, B. (2013) Dictionnaire amazighe-français: Le parler des Ayt Wirra, Moyen Atlas-Maroc, Rabat: Institut royal de la culture amazighe. 
Rabdi, L. (2004) Le parler d'Ihbachen (Kabylie Orientale, Algérie): esquisse phonologique et morphologique, Cologne: Köppe, (Berber Studies, 7).

Rabhi, A. (1994) Les particules de négation dans la Kabylie de l'Est, Etudes et documents berbères, 9, p. $139-145$.

Rabhi, A. (1996) De la négation en berbère: les données algériennes, La négation en berbère et an arabe maghrébin, Paris: L'Harmattan, p. 23-48.

Schiattarella, V. (2015) Le berbère de Siwa: documentation, syntaxe et sémantique, Thèse doctorale, Paris: Ecole Pratique des Hautes Etudes.

Schwenter, S.A. (2005) The pragmatics of negation in Brazilian Portuguese, Lingua, 115, 10, p. 1427-1456.

Souag, L. (2009) Siwa and its significance for Arabic dialectology, Zeitschrift für arabische Linguistik, p. 51-75.

Souag, L. (2017) Le parler berbère de Sokna (Lybie) à la lumière de nouvelles données, Études et Documents Berbères, 37, p. 193-203.

Souag, L. (2018) Arabic-Berber-Songhay contact and the grammaticalization of 'thing', in Manfredi S. \& M. Tosco (eds) Arabic in contact, Amsterdam: John Benjamins, p. 54-71.

Thomason, S. \& T. Kaufman (1988) Language contact, creolization, and genetic linguistics, Berkeley: University of California Press.

Van Putten, M. \& L. Souag (2015) Attrition and revival in Awjila Berber, Facebook posts as a new data source for an endangered Berber language, Corpus, 14, p. 23-58.

Willis, D., Lucas, C. \& A. Breitbarth (2013) The History of Negation in the Languages of Europe and the Mediterranean: Volume I Case Studies, Oxford: Oxford University Press Oxford.

\section{NOTES}

1. Je souhaite remercier abondamment Amina Mettouchi, Cyril Aslanov, Lameen Souag et Maarten Kossmann, pour leurs relectures et leurs commentaires sur une version précédente de ce travail.

2. L'autre directionalité, c'est-à-dire la convergence et l'emprunt de structures morphosyntaxiques de l'arabe jijélien au berbère (plutôt analysé comme la rétention d'un substrat) n'a pour l'instant fait l'objet que de quelques travaux : Kossmann 2014, Garaoun 2018. Marçais (1956) questionne également ce point à de nombreuses reprises. Les particularités de la négation en arabe jijélien n'ont toutefois pas encore été questionnés de ce point de vue.

3. Le phénomène n'est pas inconnu parmi les langues berbères, bien que l'origine des éléments relevés ne soit pas toujours claire. Un négateur préverbal la probablement emprunté à l'arabe est observé en chaoui, à Siwa, et en sanhadja de Sraïr (Lafkioui 2007 : 234-235). Les valeurs de ce négateur diffèrent selon les variétés (Kossmann 2013 :331), indiquant des origines ou des processus d'intégration de la forme emprunté différentes. Souag (2009) suggère, comme source du la en usage à Siwa, une variété d'arabe ancienne éteinte. En effet, la n'est attesté en arabe maghrébin que comme négateur verbal expressif (prohibitif ou assertif), tandis que le berbère de Siwa lui attribue un usage tout à fait canonique. Cette valeur est également partagée par le sanhadja de Sraïr, tandis que le chaoui lui préfère un usage assertif plus proche de ses valeurs en arabe maghrébin moderne (Mettouchi, 2012 : 5393). Ces résultats ont amené Kossmann (2013 : 331-332) à proposer que le la canonique attesté à Siwa et chez les Sanhadjas de Sraïr soit la 
conséquence d'une évolution du la emprunté à l'arabe maghrébin pour une valeur premièrement spécialisé (comme en chaoui) vers un usage plus canonique. Kossmann (2013: 333) signale également que l'arabe šay? " chose ", grammaticalisé en négateur postverbal dans la majorité des variétés d'arabe maghrébin est emprunté par plusieurs langues berbères dans le Rif (Ghomara, Sanhadja, Bni Iznassen etc.) et dans les oasis situées à la frontière algéro-marocaine (Figuig, Tiout etc.) qui présentent des négateurs postverbaux šay ši. Quant aux nombreuses variétés zénètes présentant un négateur postverbal $̌$, il est difficile voire impossible de déterminer s'il s'agit de l'arabe šay? ou de l'élément *kra réduit et palatalisé (ibid.). Enfin Lafkioui (2013), décrit un négateur postverbal bu en usage en rifain et dans l'arabe pratiqué à Oudja, pour laquelle l'auteure propose une origine berbère.

4. Les hypothèses concernant l'origine et la décomposition de ce marqueur sont nombreuses (Mettouchi, 2012 : 5395). Retenons Loubignac (1924) qui propose une première origine verbale de ce marqueur : tamazight du Moyen-Atlas at « être désert ». Marcy (1936:56-57) en propose une autre, un radical WR «ne pas exister/être ». Galand (1994:170-176) décompose ce morphème en un élément proprement négatif $u / w e$ en composition avec un $-r$ dont l'origine serait la préposition ar " jusqu'à », avant de lui proposer une origine nominale (ara) en 2010 puis verbale, iri « vouloir» (2011). Brugnatelli (2011) défend une origine verbale de -r. Lafkioui et Brugnatelli (2020) font dériver *(A)R/L d'un ancien auxiliaire modal.

5. Le correspondant [1] de la radicale $[r]$ est attestée pour ce négateur dans un certain nombre de variétés, en particulier parmi celles appartenant au groupe zénète (mozabite, chaoui, ouargli etc.).

6. Selon Chaker (1996). Nous ne nous entendrons pas sur la définition de ces environnements lesquels nécessiteraient une étude consacrée. Nous renvoyons cependant pour leur étude en kabyle occidental aux travaux de Mettouchi 2005, 2009, 1996.

7. Lafkioui $2007: 235$ et Gutova (communication personelle).

8. Cet usage préverbal de ula est d'un intérêt particulier, il conviendrait de vérifier si celui-ci n'est pas en lien avec la convergence sur l'arabe (cf. Kossmann 2013 : 331-332 et les débats sur la particule préverbal la).

9. D'autres parlers chaouis septentrionaux présentent une forme anic d'après Rabdi (2004:107).

10. D'après Mettouchi (communication personelle) : « celui-ci semble indiquer la complétude, il y aurait comme un parcours de toutes les situations pour dire qu'aucune n'est vraiment envisageable. "

11. Voir la synthèse de Mettouchi (2012 :5395) pour les diverses hypothèses sur ce marqueur.

12. Nous avons récemment observé l'utilisation du négateur postverbal $k a$ dans le parler de la région de Mentanou, zone frontalière entre l'aire tasahlit et chaoui des Amouchas dont le parler s'apparente plutôt au second groupe. Nous pensions jusque-là cette forme spécifique au tasahlit oriental dans les Babors. Son attestation au sud, dans un parler chaoui des Babors rend très vraisemblable l'existence de formes ressemblantes dans les parlers Tasahlit des petites confédérations situées entre l'aire du tasahlit oriental et le chaoui des Amouchas (région située entre le mont Tababort et le mont Babors).

13. Le berbère de l'atlas blidéen connaît également entre autres ce négateur postverbal (El Arifi $2016: 29)$.

14. Lafkioui $2007: 235$.

15. En effet d'après Galand (2010: 283), le pronom indéfini kra correspond à la combinaison d'un indéfini initial $k a$ avec le verbe iri vouloir: *ka yra. En tant que pronom indéfini les parlers tasahlit ne connaissent plus que la forme composées $k r a$, mais les négateurs signalés ( $k a$ et (e)k pourraient réactualiser une forme primitive $k(a)$ comme ils pourraient correspondre à des évolutions érodées phonétiquement $k r a$.

16. La forme tasahlit présente un assourdissement de la dentale sonore, trait caractéristique des parlers berbères et arabes de la Kabylie orientale. Les parlers arabes jijéliens des Babors 
occidentales présentent eux aussi cet assourdissement auprès ce verbe : țha yəțha (arabe des Aït Achour, Ziama-Mansouriah).

17. Ce qui constitue un indice de sa grammaticalisation.

18. D'après Sofiane Smaïl, ce verbe contient en kabyle occidental une certaine valeur expressive de nostalgie ou de regret.

19. Mozabite: trouver quelqu'un ou quelque chose par hasard, toucher, effleurer, se trouver quelque part par hasard, échoir à quelqu'un (Delheure 1984 :35).

20. Voir Chaker 1997, Naït-Zerrad 2005, etc.

21. Également connue pour une tendance à la grammaticalisation du négateur postverbal comme unique négateur (Khaloua, communication personelle).

22. Forme également attestée en kabyle occidental, où elle n'est utilisée que seule, comme négateur emphatique correspondant au français «pas du tout ! certainement pas!» (Mettouchi, communication personelle).

23. Si ce n'est par une influence des négateurs issus de țhu.

24. Type également représenté en berbère-Sud.

25. Au point que les auteurs s'accordent sur son ancienneté et de son caractère pan-berbère (Kossmann, 2013, Mettouchi, 2009).

26. En plus de la tasahlit occidentale des Aït Bouysef.

27. Dans certains parlers, le marquage ou non de l'accompli négatif associé à l'utilisation d'un néo-négateur est porteur de valeurs pragmatiques dans le discours.

28. Rappelons que les différents parlers tasahlit présentent plusieurs particules d'aoristes (Garaoun 2019) : $i \sim d i \sim d a \sim a \sim a d$.

29. Optatif-négatif

30. Négateur emphatique.

31. Nous avons récolté dans des corpus spontanés et dans différentes localités quelques rares occurrences de néo-négateurs préverbaux accompagnés de négateurs postverbaux. Ces usages ne nous ont toutefois pas paru grammaticalisés, nous les avons analysés comme le résultat d'interférence syntaxique entre le type de négation déclaratif à négateur préverbal $u l+N E G 2$. Il reste pour autant tout à fait possible que ce type d'usage se grammaticalise dans le futur.

32. En excluant les variétés sahariennes et hassaniya.

33. Formes à l'origine de différentes variantes selon les parlers : baci, mac, mači, etc.

34. Contrairement aux négateurs de négation modale/non-déclarative de fond berbère, présentant eux aussi une position préverbale, mais offrant toujours la possibilité d'une association à un négateur ou complémenteur postverbal.

35. Le négateur assertif préverbal ma est aussi connu en kabyle, le chaoui lui préfère la également emprunté (Mettouchi, 2012 : 5393).

36. En arabe maghrébin, les énoncés assertifs font souvent appel au registre religieux lorsqu'ils ne contiennent pas des formules directement empruntées à la langue coranique.

37. Ces données viennent alimenter le débat entretenu par Souag (2009) et Kossmann (2013 : 331-332) concernant l'origine du développement du négateur emprunté la dans les différentes langues berbères qui l'attestent pour un usage emphatique ou canonique. En effet, dans un grand nombre de variétés d'arabe maghrébin, ce dernier négateur apparait surtout dans le contexte de la négation assertive venant remplacer le modèle de négation déclarative à deux éléments $m a$ préverbal $+\check{s}(i / \bar{a} y)$ postverbal.

38. Nous pensons spécialement aux érosions de l'accompli négatif et de l'attraction des satellites du verbe qui, bien qu'attestés dans un grand nombre de variétés, semblent présenter des stades particulièrement avancés dans les variétés de communautés bilingues berbère-arabe maghrébin. Il conviendrait de procéder à des études comparatives de ces érosions à l'échelle pan-berbère en les comparant au données sociolinguistiques / situations de contact locales. 
39. Ce qui correspond au facteur de recherche d'expressivité comme moteur du changement dans les développements accusés par les systèmes de la négation berbères (Galand 1994).

\section{RÉSUMÉS}

Le berbère tasahlit est une variété de berbère-Nord pratiquée dans la Kabylie des Babors, dans le nord-est algérien (200 000 locuteurs). Au contact d'une variété d'arabe préhilalien depuis plus d'un millénaire (l'arabe jijélien dont l'émergence date $d u 7^{\mathrm{e}}$ siècle), les deux langues se sont profondément interpénétrées. Nous regarderons ici les conséquences de ce contact en berbère, au niveau de la négation verbale en énoncé déclaratif. En effet, celle-ci présente parmi les différents parlers tasahlit des négateurs issus de grammaticalisations de verbes d'origine arabe. Nous décrirons ces négateurs et leurs usages, et tenterons de démontrer comment, dans ce contexte de contacts, emprunts et convergences riment avec complexité et diversité.

Tasahlit is a Northern Berber language spoken in the Babor Mountains, off the northeast coast of Algeria. It is spoken close to the linguistic area of Jijel Arabic: a Maghrebi Arabic variety considered to be "pre-Hilalian", that is to say originated from the first wave of Arabization of North Africa ( $7^{\text {th }}-9^{\text {th }}$ century). Both of these languages belong to the Afro-Asian phylum (formerly Hamito-Semitic).

This Berber-Arabic contact situation at the heart of Eastern Kabylia is the source of deep convergence in the two systems, at different linguistic levels. The morphosyntax of negation is one of the areas that are most affected by this contact in Tasahlit. The developments that we noted, more or less appropriable to convergence, by no means simplified the whole but rather caused a multiplication of forms and rules.

Negation is one of the most studied morphosyntactic areas in both Berber languages and Maghrebi Arabic (Galand, 1994; Chaker \& Caubet, 1996; Mettouchi, 1996, 2009; Lucas, 2007, 2013; Lafkioui \& Brugnatelli, 2017, 2020), and it has often been discussed in relation to Berber-Arabic convergence (Brugnatelli, 2014; Lafkioui \& Brugnatelli, 2020; Kahlouche, 2000; Mettouchi, 2012; Kossmann, 2013; Lucas, 2007, 2009, 2013, 2017; Souag, 2018).

In this article, convergence is understood as a trans-linguistic change characterized by approximation between structures of different linguistic systems. This convergence is mainly observable in extensive contact situations (Thomason \& Kaufman, 1988). The old and intense contact between Maghrebi Arabic and Berber is sometimes part of convergence dynamics, which vary in intensity from one contact area to another (Kossmann, 2013). Observable differences between Arabic-Berber contact situations provide us with valuable data about the diversity of their sociohistorical conditions (Souag, 2009).

Negation presents a certain number of characteristics in Tasahlit: this language has the highest diversity of negation markers in Berber. Each of these varieties certifies the use of a series of preverbal and postverbal negators that are sometimes specific to a certain language. Among the latter, multiple borrowings from Arabic continue to be grammaticalized as markers which were added to the plurality of preexisting negation markers of Berber origin. Moreover, the syntax of negation in Berber presents variability in particular developments depending on the Berber language it concerns. Again, the different Tasahlit varieties show original results, marked by the erosion of the old system, more or less preserved.

In the present work, we need to describe "neo-negators", their origins through their 
grammaticalization path, as well as the changes that they cause on the morphosyntax of negation in Tasahlit Berber. This analysis is conducted by interrogating convergence between Northern Berber and Maghrebi Arabic.

The Tasahlit Berber area $(\approx 200,000$ speakers) comprises close to fifteen varieties, most of which were never described. In March 2020, we investigated the issue of negation in Tasahlit through fieldwork, in addition to the data gathered since 2018 through multiple other fieldworks. During this investigation, we held some taped interviews with speakers originating from 9 Tasahlitspeaking confederations. The gathered data was then compared to that of other Berber languages, of Maghrebi Arabic, of Levantine Arabic, and of Classical Arabic.

We have prepared the etymons of negation markers of Berber origin collected from the different varieties, along with two series of verbal negators grammaticalized through elements borrowed from Arabic: thu and $x t u$. The first series comes from an original grammaticalization of the Arabic verb Padha "make/become in the morning (duhậ)".

The second one is more predictable, since its value XṬ "lack" is already negative. We have shown that these grammaticalizations of words borrowed as modal/non-declarative verbal negation markers occurred through their use in emphatic or nominal and non-modal negation.

These neo-negators cause or complement multiple morphosyntactic changes. These transformations result in forms that are closer to Arabic than to conservative Berber. This issue deserves to be studied from the perspective of convergence, even though most of the time internal motivations could be raised. We have noted that the use of neo-negators resulted in:

- The reinforcement of the tendency to drop the negative perfect.

- The erosion of the phenomenon of attraction of satellite verbs.

- The emergence of the possibility of negatively flexing a statement in aorist + particle.

- The reinforcement of verbal negation through one unique preverbal negator.

Multiple noted phenomena correspond to simplifications of the system caused by an exhaustion of specific markings. In other Berber varieties, many syntactic evolutions caused by the use of neo-negators are known at different degrees, but their combination in Tasahlit constitutes a particularly original case. We believe that, in the event that these neo-negators totally replace their pan-berber correspondents, and in one that the syntactic erosions currently underway come to an end, one could speak about a typological shift of negation in Tasahlit Berber, marked by an approximation with data from Maghrebi Arabic.

\section{INDEX}

Mots-clés : Berbère, arabe, contact, tasahlit, convergence

Keywords : Berber, Arabic, contact, Tasahlit, convergence

\section{AUTEUR}

\section{MASSINISSA GARAOUN}

Ecole Pratique des Hautes Etudes (EPHE), Laboratoire des Langues Langages et Cultures d'Afrique (LLACAN)

massinissagaraoun@gmail.com 